\title{
MicroRNA-21 (Mir-21) Promotes Cell Growth and Invasion by Repressing Tumor Suppressor PTEN in Colorectal Cancer
}

\author{
Yiying Wu ${ }^{a, b}$ Yi Song ${ }^{c}$ Yao Xiong ${ }^{b}$ Xiaodong Wang ${ }^{d} \quad K \mathrm{Xu}^{\mathrm{b}}$ Bin $\mathrm{Han}^{\mathrm{b}}$ \\ Yang Bail ${ }^{\mathrm{b}}$ Li Lid ${ }^{\mathrm{d}}$ Yuanyuan Zhang ${ }^{\mathrm{b}}$ Liming Zhou
}

${ }^{a}$ Department of Pharmacology, School of Pharmacy, Chengdu Medical College, Chengdu, bepartment of Pharmacology, West China School of Preclinical and Forensic Medicine, Sichuan University, Chengdu, 'Department of pharmacy, West China Hospital, Sichuan University, Chengdu, Sichuan, dDepartment of surgery, West China Hospital, Sichuan University, Chengdu, Sichuan, China

\section{Key Words}

Colorectal carcinoma • MiR-21 • PTEN • Proliferation • Invasiveness

\begin{abstract}
Background/Aims: MicroRNA-21 (miR-21) has been demonstrated to play an important role in carcinogenesis; however, its mechanism of action in colorectal cancer (CRC) has not been fully elucidated. The aim of the present study was to explore the oncogenic function of miR-21 and its molecular mechanism in CRC. Methods: A total of 105 paired tumor and tumor-adjacent normal tissue specimens from CRC patients and two CRC cell lines (HCT116 and SW480) were studied. The protein and mRNA expression levels of PTEN and miR-21 were examined using western blot analysis and real-time reverse transcription-PCR (qRT-PCR). Furthermore, we transfected CRC cells with different combinations of ectopic-expression vector or shRNA expression vector of miR-21 and phosphatase and tensin homolog (PTEN) to modulate the expression levels of miR-21 and PTEN respectively, and then analyzed the phenotypic alterations of CRC cells. Tumorigenesis was also evaluated by xenografting HCT116 cells into nude mice. Results: In this study, we showed that miR-21 expression was significantly up-regulated in CRC compared to that in normal tissues. Patients with advanced Tumor-Node-Metastasis (TNM) stage, lymph node metastasis, local invasion and higher serum carcinoembryonic antigen (CEA) levels displayed significantly high expression of miR-21. The PTEN protein level in CRC tissues and cells was inversely correlated with miR-21 expression. Furthermore, the transfection of CRC cells with pre-miR-21 could inhibit apoptosis and promote cellular proliferation, invasion, cell cycle progression and growth of xenografts in nude mice, whereas the transfection of miR-21-specific shRNA resulted in the opposite phenomena. In addition, silencing or elevating PTEN protein could partially reverse the effect of miR-21-specific shRNA or pre-miR-21 on apoptosis, cell cycle distribution, and invasion of CRC cells. Moreover, over-expression or knockdown of miR-21 altered the protein expression $\mathrm{Y}$. Wu and Y. Song contributed equally to this work.
\end{abstract}

Liming Zhou

and Dr Yuanyuan Zhang

KARGER
Department of Pharmacology, West China School of Preclinical and Forensic Medicine, Sichuan University, Chengdu, Sichuan, (China)

Tel. (086) 028-85503767; E-Mail zhou108@163.com/sarahyyzhang@hotmail.com 
of PTEN and phosphorylated Akt (p-AKT). Conclusion: miR-21 can modulate the malignant phenotypes such as proliferation, anti-apoptosis, cell cycle progression and invasion of CRC cells by down-regulating PTEN protein expression. The results of study might improve our understanding of the regulatory mechanism of miR-21 and provide useful targets and approaches for the clinical diagnosis and therapy of CRC.

(C) 2017 The Author(s)

Published by S. Karger AG, Basel

\section{Introduction}

Colorectal cancer (CRC) is one of the most common malignancies and a leading cause of cancer-related death worldwide [1]. Surgical resection and chemoradiotherapy are still the primary therapeutic approaches for CRC; however, $25 \%-30 \%$ of patients are diagnosed too late to be eligible for resection. Moreover, 30\%-50\% patients with CRC will develop recurrence and metastasis, with more than $90 \%$ of the recurrences in the first 5 years following surgery. The outcomes of patients diagnosed with CRC remain quite poor $[2,3]$. Analysis of the genome and proteome of CRC cells has demonstrated that focusing on molecular heterogeneity in colorectal carcinogenesis may provide a viable target and approach for the clinical diagnosis and therapy of CRC. Numerous studies and resources have been dedicated to elucidate the molecular mechanisms of CRC. However, the molecular pathogenesis of CRC is complicated and remains unclear.

MicroRNAs (miRNAs) are endogenously expressed short, noncoding, single-stranded RNAs of 18-25 nucleotides in length. In general, miRNAs are transcribed as a cluster called the pri-miRNA complex, which is cleaved in the nucleus to form the pre-miRNA that is then translocated to the cytoplasm to undergo final maturation into a functional miRNA. miRNAs can modulate gene expression at the post-transcriptional level by targeting mRNAs for translational repression or degradation. Many studies have demonstrated that miRNAs play key roles in the regulation of cellular growth, differentiation, proliferation, and apoptosis [4]. Furthermore, it has been shown that miRNAs can function as tumor suppressors or oncogenes and are involved in various biological processes such as tumor initiation, tumor progression and drug resistance in various types of cancer, including CRC [5-10]. miR21 , a putative oncogene, is most frequently over-expressed in various malignancies, and it has been implicated in multiple malignancy-related processes including uncontrolled cell proliferation, inhibition of apoptosis and promotion of invasion and metastasis [1113]. According to recent reports, several significant targets of miR-21 associated with malignancy have been experimentally validated, such as PTEN [14-20], programmed cell death 4 (PDCD4) [21-23], reversion-inducing cysteine-rich protein with Kazal motifs (RECK) [24], forkhead box 01 (FOXO1) [25], RhoB [26] and Cdc25a [27], and these targets may exert different effects on tumorigenesis. Among them, the PTEN protein was reported to be frequently silenced in CRC, and it has been shown to suppress tumor formation by inhibiting the PI3K/AKT pathway $[28,29]$. Therefore, miR-21 might act as an important oncogene by regulating PTEN as one of its several target genes and thus may be a useful biomarker for the diagnosis and treatment in CRC.

Previous studies have shown that miR-21 is up-regulated in CRC and functions as an oncogene. However, the biological roles and exact mechanisms of miR-21 in the carcinogenesis and development of CRC remain elusive. The aim of the present study was to explore the oncogenic function of miR-21 and its molecular mechanisms in CRC. In this study, we determined the expression of miR-21 and PTEN protein in human CRC tissues and correlated them with clinicopathological features of CRC patients. Subsequently, in order to evaluate the role of miR-21 in the proliferation, apoptosis, cell cycle distribution and invasion of CRC cells, as well as its effect on target genes, the recombinant ectopic-expression and short hairpin RNA (shRNA)-expression plasmids of miR-21 and PTEN were constructed, and transfected individually or together into the CRC cell lines HCT-116 and SW480. Finally, we demonstrated the effects of miR-21 on tumor formation and the regulatory mechanism of miR-21 in vivo in a human CRC model using nude mice. The results of this study may improve 


\section{Cellular Physiology Cell Physiol Biochem 2017;43:945-958

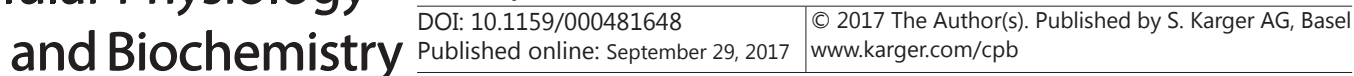 \\ Wu et al.: Mir-21 and Colorectal Cancer}

our understanding of the regulatory mechanism of miR-21 and provide useful targets and approaches for the clinical diagnosis and therapy of CRC.

\section{Materials and Methods}

\section{Patients and tissue samples}

Paired CRC and adjacent non-tumor tissues were obtained with informed consent from 105 patients who underwent surgical resection of primary CRC between 2010 and 2011 at the West China Hospital, Chengdu, China. The median age of the patients was 60 years, ranging from 30 to 85 . All samples were immediately frozen in liquid nitrogen after resection and stored at $-80^{\circ} \mathrm{C}$ for further detection. Both tumor and non-tumor samples were confirmed by pathological examinations. Patient clinicopathologic characteristics including gender, age, tumor site, local invasion, differentiation, lymph node metastasis and serum CEA levels were obtained from surgical and pathological records from the hospital, and all the data were analyzed anonymously. This study was carried out with the approval from the Medical Ethical Committee of West China School.

\section{Cell culture}

Human colorectal cancer cell lines HCT-116 and SW480 were obtained from the Key Laboratory of Transplant Engineering and Immunology of the Ministry of Health, West China Hospital Sichuan University. The cells were cultured in RPMI-1640 (Gibco, USA) supplemented with $10 \%$ fetal bovine serum (Minhai Bio-engineering, China), $100 \mathrm{U} / \mathrm{mL}$ penicillin and $100 \mu \mathrm{g} / \mathrm{mL}$ streptomycin (Huabei Pharmaceuticals Ltd, China), and kept at $37^{\circ} \mathrm{C}$ in a humidified incubator with $5 \% \mathrm{CO}_{2}$.

\section{Plasmid constructs and transfection}

To construct the recombinant ectopic-expression and shRNA-expression plasmids of miR-21 and PTEN, the corresponding gene sequences encoding pre-miR-21 and PTEN were amplified from HCT-116 cells and cloned into the pEGFP-N1 vector (Invitrogen, USA), and the shRNA sequences of miR-21 and PTEN, as well as the scrambled control sequences, were designed and cloned into the Psilencer4.1-CMV vector (Invitrogen, USA). Consequently, the ectopic-expression plasmids pEGFP-pre-miR-21 (pE-miR-21) and pEGFP-PTEN (pE-PTEN) for the over-expression miR-21 and PTEN, respectively, and the shRNA-expression plasmids Psilencer4.1-miR-21-shRNA (miR-21 shRNA) and Psilencer4.1-PTEN-shRNA (PTEN shRNA) to knock down miR-21 and PTEN, respectively, were constructed. Then, in order to explore the functional role of miR-21 in CRC, the ectopic-expression or shRNA-expression plasmids for miR-21 and PTEN was transfected alone or together into the CRC cell line HCT-116 or SW480 using Lipofectamine 2000 (Invitrogen, USA) according to the manufacturer's instructions. The expression of miR-21 and PTEN was verified using real-time PCR $48 \mathrm{~h}$ after transfection. Stable cell lines (HCT-116 cells transfected with null vector pEGFP-N1, pE-miR-21, miR-21 shRNA or scrambled sequence control) were selected with $600 \mu \mathrm{g} / \mathrm{ml}$ of G418, and the expression of miR-21 was also confirmed using real-time PCR.

\section{RNA isolation and real-time PCR}

Total RNA was isolated from cells and tissues using TRIzol reagent (Invitrogen, USA) according to manufacturer's instructions. One microgram of total RNA was reverse-transcribed to cDNA using the RevertAid $^{\mathrm{TM}}$ First Strand cDNA Synthesis Kit (Fermentas, USA). miR-21 and the reference gene U6 were amplified usinggene-specificstem-loop primers. The cDNA wasused for the quantification of miR-21 andPTEN. Real-time PCR was performed using SYBR Premix Ex Taq ${ }^{\text {TM }}$ (Takara, Japan) according to the manufacturer's protocol. The reactions were carried out using an Applied Biosystems 7500 Fast Real-time PCR system (Applied Biosystems Inc., USA). For estimating the differential expression of miR-21 and PTEN mRNA, the Ct values were normalized using U6 and GAPDH mRNA, respectively, as internal controls. Primer sequences for miR-21 (RT-primer: 5'-GTCGTATCCAGTGCAGGGTCCGAGGTATTCGCACTGGATACGACTCAACA-3'; forward: 5'-CGGCGGTAGCTTATCAGACTGATGT-3'; reverse: 5'-GTGCAGGGTCCGAGGT-3'), U6 (RTprimer: 5'-CGCTTCACGAATTTGCGTGTCAT-3'; forward: 5'-CTCGCTTCGGCAGCACA-3'; reverse: 5'-AACGCTTCACGAATTTGCGT-3'), PTEN (forward: 5'-ACCATAACCCACCACAGC-3'; reverse: 5'-CAGTTCGTCCCTTTCCAG-3'), and GAPDH (forward: 5'-ACGGATTTGGTCGTATTGGG-3'; reverse: 


\section{Cellular Physiology Cell Physiol Biochem 2017;43:945-958 \begin{tabular}{ll|l} 
and Biochemistry $10.1159 / 000481648$ & $\begin{array}{l}\text { C } 2017 \text { The Author(s). Published by S. Karger AG, Basel } \\
\text { www.karger.com/cpb }\end{array}$ \\
\hline
\end{tabular}}

Wu et al.: Mir-21 and Colorectal Cancer

5'-CGCTCCTGGAAGATGGTGAT-3') were synthesized by Baosheng biotechnology companies (China). The relative expression level was calculated using the $2^{-\Delta \Delta \mathrm{Ct}}$ method. All reactions were performed in triplicate within the same PCR run.

Western blot

Cells or tissues were harvested and lysed in lysis buffer on ice for $30 \mathrm{~min}$. The lysate was centrifuged at $12,000 \mathrm{rpm}$ at $4^{\circ} \mathrm{C}$ for $15 \mathrm{~min}$, and protein concentrations were measured with a protein assay kit (Bio-Rad, USA). Equal amounts of proteins were electrophoresed using SDS-PAGE gels and subsequently transferred to PVDF membranes (Bio-Rad laboratories, USA). After blocking with 5\% bovine serum albumin (BSA) at $4^{\circ} \mathrm{C}$ overnight, the membranes were incubated with antibodies against PTEN, p-AKT and $\beta$-actin (Santa Cruz, USA) in TBST at room temperature for $2 \mathrm{~h}$. After washing three times with TBST, the membranes were incubated with peroxidase-conjugated secondary antibodies (Santa Cruz, USA) for $1 \mathrm{~h}$, and then the protein signals were detected with electrochemiluminescence (ECL) western blot detection reagents (Beyotime, China). Expression levels of the proteins were compared to the control based on the relative intensities of the bands. Band density was quantified using the Bio-Rad Quantity One v4.62 software.

\section{Cell proliferation assay}

Cell proliferation was determine using the 3-(4, 5-dimethylthiazol-2-yl)-2, 5-diphenyltetrazolium bromide (MTT; Sigma, USA) assay. Cells were plated in 96 -well plates at $5 \times 10^{3}$ cells per well and transfected with recombinant plasmids or non-specific control plasmids. After incubation for $24 \mathrm{~h}, 20 \mu \mathrm{l}$ of the MTT solution was added to each well and incubated for $4 \mathrm{~h}$ at $37^{\circ} \mathrm{C}$. The supernatant was removed, and the formazan crystals was dissolved in $100 \mu \mathrm{l}$ DMSO. The absorbance of the suspension was measured at 570 $\mathrm{nm}$ at $24,48,72,96 \mathrm{~h}$ after transfection.

\section{Flow cytometric analysis}

Cell cycle distribution and apoptosis were evaluated using flow cytometric analysis. Briefly, cells were harvested $48 \mathrm{~h}$ after transfection. For cell cycle analysis, the transfected cells were washed with cold PBS and fixed in $70 \%$ ethanol overnight at $4^{\circ} \mathrm{C}$. The next day, the cells were incubated with $0.01 \%$ RNase at $37^{\circ} \mathrm{C}$ for $10 \mathrm{~min}$ and then stained with $50 \mu \mathrm{g} / \mathrm{mL}$ propidium iodide (PI) at $4^{\circ} \mathrm{C}$ in the dark for $20 \mathrm{~min}$. Cell cycle distribution was examined with a FACSCalibur Flow Cytometer (Becton Dickinson, USA). Meanwhile, the transfected cells were dual-stained with the viability dye 7-amino-actinomycin D (7-AAD) and Annexin V-APC using an Annexin V-APC/7-AAD Apoptosis Detection Kit (KaiJi, China) following the manufacturer's protocol. The stained cells were immediately analyzed using flow cytometry.

\section{Cell invasion assay}

Cell invasion was examined using 24-well Matrigel-coated transwell chambers (Becton Dickinson, USA). In brief, cells were washed with PBS and resuspended at $1 \times 10^{5}$ cells $/ \mathrm{mL}$ in serum-free medium 24 $\mathrm{h}$ after transfection. Then, $0.2 \mathrm{ml}$ cell suspension was added to the upper chamber, and $0.5 \mathrm{ml}$ medium containing 10\% FBS was added to the lower chamber. After incubation for $24 \mathrm{~h}$, all non-invaded cells were removed from upper surface of the filters, and the invaded cells were fixed and stained with crystal violet solution. The experiments were performed in triplicate, and the invaded cells were counted under a microscope $(400 \times)$ in five different fields per filter.

\section{Tumor xenografts}

Forty 4- to 5-week-old female athymic nude mice were divided into 5 groups as follows: pE-miR-21, miR-21 shRNA, scrambled control, null vector control, and negative control (inoculated with untreated HCT-116 cells). Stably transfected HCT-116 cells were resuspended in PBS and subcutaneously inoculated into the right axilla of mice $\left(1 \times 10^{7}\right.$ cells/mouse). Mice were maintained in a pathogen-free environment. From day 4, tumor volume (V) was measured every other day and calculated according to the formula $\mathrm{V}$ $\left(\mathrm{mm}^{3}\right)=\mathrm{ab}^{2} / 2$, where $\mathrm{a}$ is the largest superficial diameter, and $\mathrm{b}$ is the smallest superficial diameter.

All mice were euthanized with pentobarbital sodium 28 days after inoculation, and the transplanted tumors were excised and weighed. Animal handling and experimental procedures were approved by the Animal Ethics Committee of Sichuan University. 


\section{Statistical analysis}

Statistical Package for Social Sciences version 13.0 (SPSS Inc, Chicago, IL) was used for standard statistical analyses. Values were presented as the mean \pm standard error of mean (SEM). The difference in miR-21 expression between CRC and matched normal tissues were examined using the Wilcoxon matched-pairs signed-ranks test. Nonparametric Wilcoxon-Mann-Whitney test was used to analyze the association between miR-21 expression level and various clinicopathologic characteristics. The correlation between PTEN and miR-21 was assessed using Spearman's rank-order correlation. Multiple group were analyzed using one-way ANOVA for subsequent individual group comparisons. A value of $\mathrm{P}<0.05$ was considered statistically significant.

\section{Results}

miR-21 over-expression is associated with clinicopathological features of CRC patients

The expression of miR-21 in 105 paired
Table 1. Comparison of miR-21 expression levels in different clinicopathologic features in CRC. a Median of relative expression; A value of $\mathrm{p}<0.05$ was considered statistically significant

\begin{tabular}{lccc}
\hline & $\mathrm{N}$ & $\mathrm{miR}^{2} 1^{\mathrm{a}}$ & $\mathrm{P}$ value \\
\hline Age & & & \\
$\leq 60$ & 57 & 3.14 & 0.834 \\
$>60$ & 48 & 3.43 & \\
Gender & & & \\
male & 58 & 3.64 & 0.920 \\
female & 47 & 3.92 & \\
$\begin{array}{l}\text { Location } \\
\text { colon }\end{array}$ & & & \\
rectum & 19 & 2.81 & 0.210 \\
TNM stage & 86 & 4.16 & \\
$\begin{array}{l}\text { I-II } \\
\text { III-IV }\end{array}$ & 57 & 1.69 & 0.000 \\
$\begin{array}{l}\text { Differentiation } \\
\text { poor }\end{array}$ & 58 & 7.22 & \\
moderate-well & & & \\
CEA & 30 & 7.54 & 0.005 \\
$\begin{array}{l}\leq 3.4 n g / m l \\
>3.4\end{array}$ & 75 & 2.88 & \\
Lymph node metastasis & & & \\
negative & 54 & 0.39 & 0.000 \\
positive & 59 & 1.75 & \\
Local invasion & 46 & 7.22 & 0.000 \\
T1+T2+T3 & & & \\
T4 & 50 & 1.72 & 0.006 \\
\hline
\end{tabular}
CRC and corresponding normal tissues was measured using real-time PCR. Compared to the normal tissues, we found significantly higher miR-21 expression of up to 3.45-fold in CRC $(\mathrm{P}<0.01)$. The median gene expression of miR-21 in the CRC samples was 5.03, and the median gene expression in the normal mucosa tissue was 1.22 .

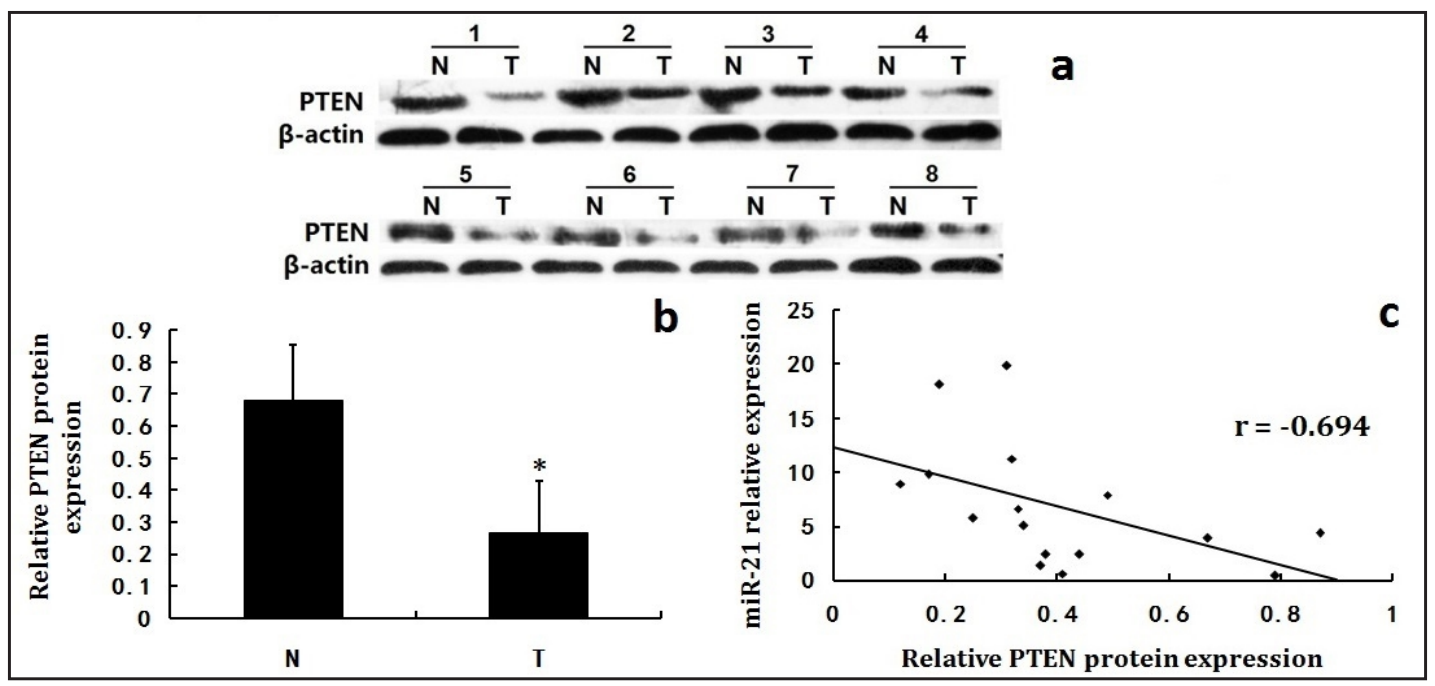

Fig. 1. Expression of PTEN protein in CRC tissues Sixteen pairs of matched CRC specimens were randomly selected for the determination of expression levels of PTEN protein. (a) Representative blots showing PTEN protein levels in 8 pairs of tumor tissues and matched non-tumor tissues. (b) Relative expression of PTEN protein in CRC tissues and matched non-tumor tissues. PTEN protein expression was detected using western blotting and normalized to $\beta$-actin protein expression. ${ }^{*} \mathrm{P}<0.05$ vs non-tumor tissue. N, non-tumor tissue; T, tumor tissue. (c) Correlation between miR-21 expression and PTEN protein levels in CRC tissues (Pearson correlation coefficient $=-0.694,{ }^{*} \mathrm{P}<0.01$ ). 
Furthermore, miR-21 expression was significantly higher in patients with advanced clinical TNM stage, poor differentiation, deeper invasion, positive lymphatic metastasis and high level of serum CEA. However, non-significant differences were observed in sex, age and tumor location. The patient characteristics along with the relative expression of miR-21 in the CRC samples obtained using real-time PCR are shown in Table 1. miR-21 expression was associated with TNM stage $(\mathrm{P}<0.01)$, histological differentiation $(\mathrm{P}<0.01)$, depth of invasion $(\mathrm{P}<0.01)$, lymph node metastasis $(\mathrm{P}<0.01)$ and serum CEA level $(\mathrm{P}<0.01)$ but not sex, age and tumor location.

\section{PTEN protein level is inversely correlated with miR-21 expression in CRC tissues}

To explore miR-21 target genes, we examined the protein expression of the tumor suppressor gene PTEN using western blotting. Among the 105 pairs of matched CRC specimens, 16 pairs were chosen for the evaluation of the correlation between miR-21 levels

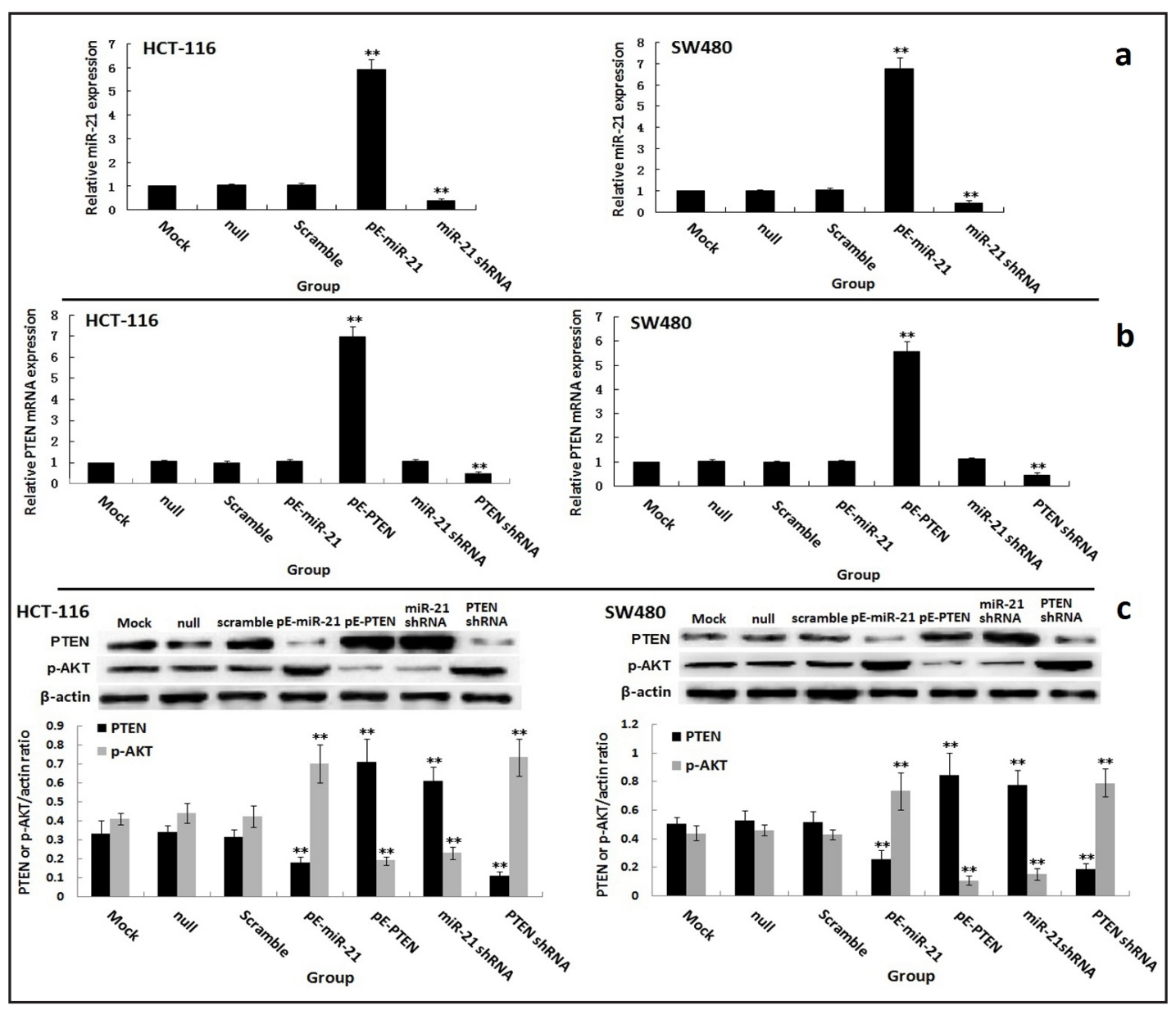

Fig. 2. miR-21 regulates PTEN expression and influences the phosphorylation of AKT Cells were transfected with pE-miR-21, miR-21 shRNA, scramble sequences plasmid (scramble), null vector control (null) or blank control containing culture medium (Mock). Cell lysates were obtained $48 \mathrm{~h}$ after transfection for analysis. (a) The expression of miR-21 in transfected cells was examined using real-time PCR. The results were normalized to U6 expression and expressed as fold change relative to those in Mock. (b) The expression of PTEN mRNA in transfected cells was examined using real-time PCR. The results were normalized to GAPDH expression and expressed as fold change relative to those in Mock. (c) The expression of PTEN protein and the phosphorylation of AKT in transfected cells were examined using western blot analysis. The results were normalized to $\beta$-actin protein expression and expressed as fold change relative to those in Mock. The band intensity was analyzed with Bio-Rad Quantity One v4.62 software. All data are representative of three independent experiments. ${ }^{* *} \mathrm{p}<0.01$ vs Mock.

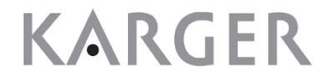


and PTEN protein expression. In comparison with the adjacent non-tumor tissues, the level of PTEN protein was significantly lower in tumor tissues as shown in Figure $1 \mathrm{a}$ and $1 \mathrm{~b}$. Moreover, we found a significant inverse correlation between the levels of miR-21 and PTEN protein (Fig. 1c) $(\mathrm{r}=-0.694, \mathrm{P}<0.01)$.

miR-21 regulates the expression of PTEN and phosphorylation of AKT

To further explore whether PTEN expression is regulated by miR-21 in CRC cells, we analyzed the expression of miR-21 and PTEN in HCT-116 and SW480 cells transiently transfected with pE-miR-21, miR-21 shRNA, scrambled sequences (scramble), null vector control (null) or blank control containing culture medium (Mock). As shown in Fig. 2a and $2 c$, the elevation or reduction of endogenous miR-21 levels with pE-miR-21 or miR-21 shRNA plasmids, respectively, resulted in a corresponding decrease or increase in PTEN protein expression relative to that in the control cells. However, there was no change in PTEN mRNA levels (Fig. 2b). As p-AKT is an important downstream target of PTEN, the changes in p-AKT was also simultaneously examined. As shown in Fig. 2c, the phosphorylation of Akt was also concomitantly increased or decreased in response to pE-miR-21 or miR-21 shRNA, which mimicked the effect of PTEN shRNA or pE-PTEN.

In addition, the regulation of PTEN by miR-21 was further confirmed in CRC xenograft tumors in nude mice. Consistent with the in vitro data, miR-21 reduced the protein expression of PTEN and elevated the phosphorylation of Akt, but the mRNA level of PTEN in tumors was not changed (Fig. 3).

\section{miR-21 promotes proliferation in CRC cell lines}

The MTT assay was used to evaluate the effect of miR-21 on cell proliferation. The results showed that the over-expression of miR-21 with pE-miR-21 plasmid could promote

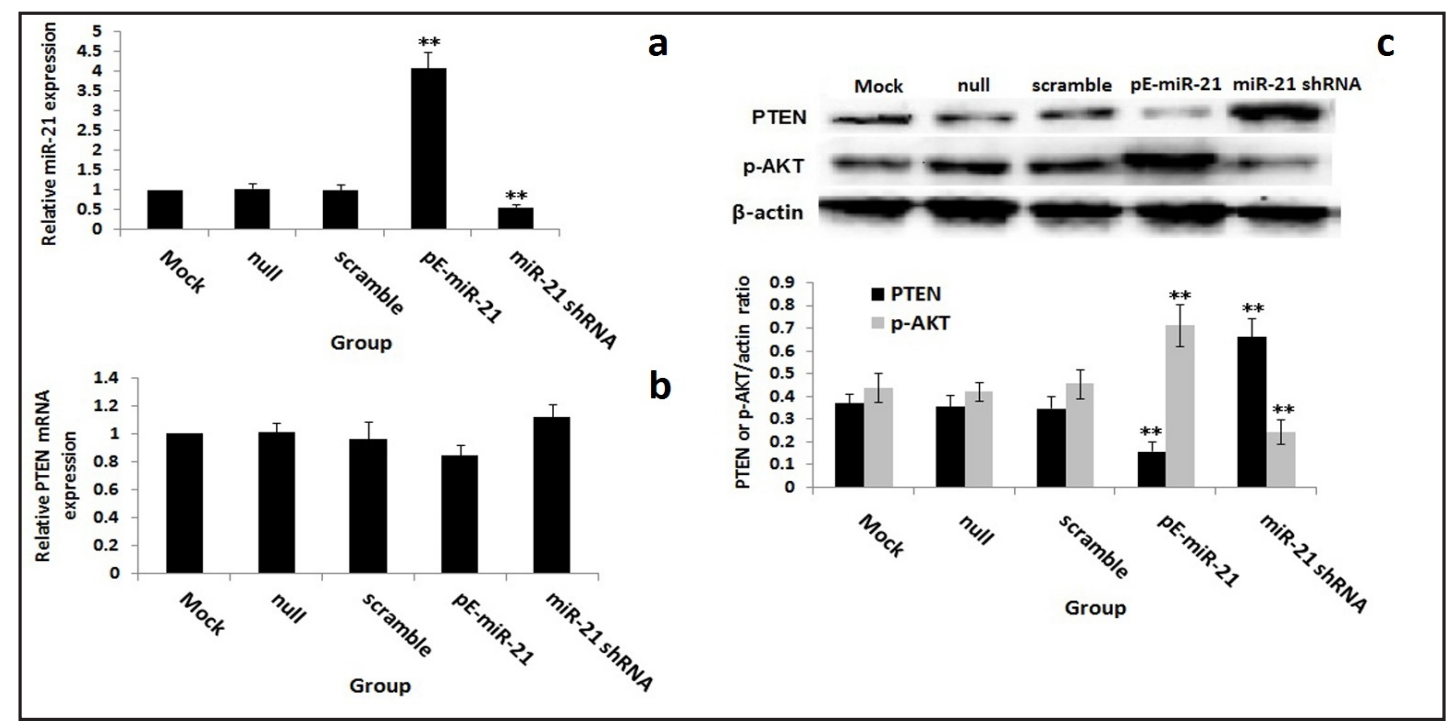

Fig. 3. miR-21 inhibits PTEN protein expression in xenograft tumors Stably transfected HCT-116 cells were subcutaneously inoculated into the axilla of nude mice to establish a CRC xenograft model. The transplanted tumors were excised for analysis 28 days after inoculation. (a) The expression of miR-21 in xenograft tumors was examined using real-time PCR. The results were normalized to U6 expression and expressed as fold change relative to those in the negative control. (b) The expression of PTEN mRNA in xenograft tumor was examined using real-time PCR. The results were normalized to GAPDH expression and expressed as fold change relative to those in the negative control. (c) The expression of PTEN protein and the phosphorylation of AKT in xenograft tumors were examined using western blot analysis. The results were normalized to $\beta$-actin protein expression and expressed as fold change relative to those in the negative control. The band intensity was analyzed using Bio-Rad Quantity One v4.62 software. All data are representative of three independent experiments. ${ }^{* *} \mathrm{p}<0.01$ vs negative control.

\section{KARGER}


Fig. 4. Growth curve of transfected cells HCT-116 and SW480 cells were transfected with pE-miR-21, miR-21 shRNA, scramble, null or Mock. Relative cell growth was determined at indicated times using cell proliferation assay. Data are presented as the mean $\pm \mathrm{SD}$ of three independent experiments.
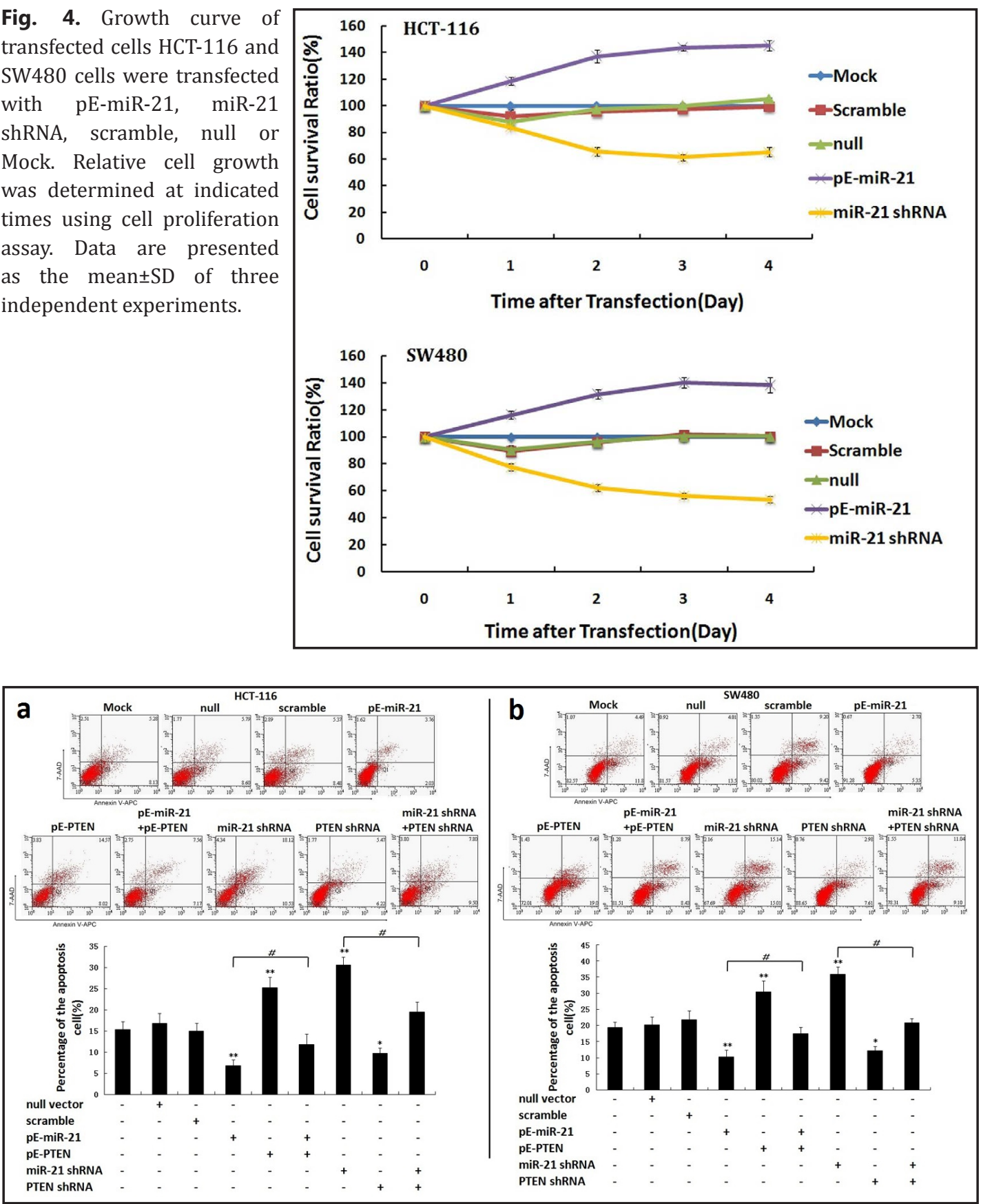

Fig. 5. miR-21 regulates apoptosis of CRC cells through PTEN Recombinant plasmids were transfected alone or together into CRC cells. Forty-eight hours after transfection, the cells were dual-stained with 7-AAD/ Annexin V-APC and analyzed using flow cytometry. Percentage of apoptotic cells is shown in the histogram. (a) Apoptosis rate of HCT-116 cells after transfection. (b) Apoptosis rate of SW480 cells after transfection. All data are representative of three independent experiments. ${ }^{*} \mathrm{p}<0.05,{ }^{* *} \mathrm{p}<0.01$ vs blank control (Mock). ${ }^{\#} \mathrm{p}<0.05$ vs cells transfected with $\mathrm{pE}-\mathrm{miR}-21$ or miR-21 shRNA.

the proliferation of HCT-116 and SW480 cells. Conversely, the suppression of miR-21 with shRNA could inhibit cell proliferation (Fig. 4). 


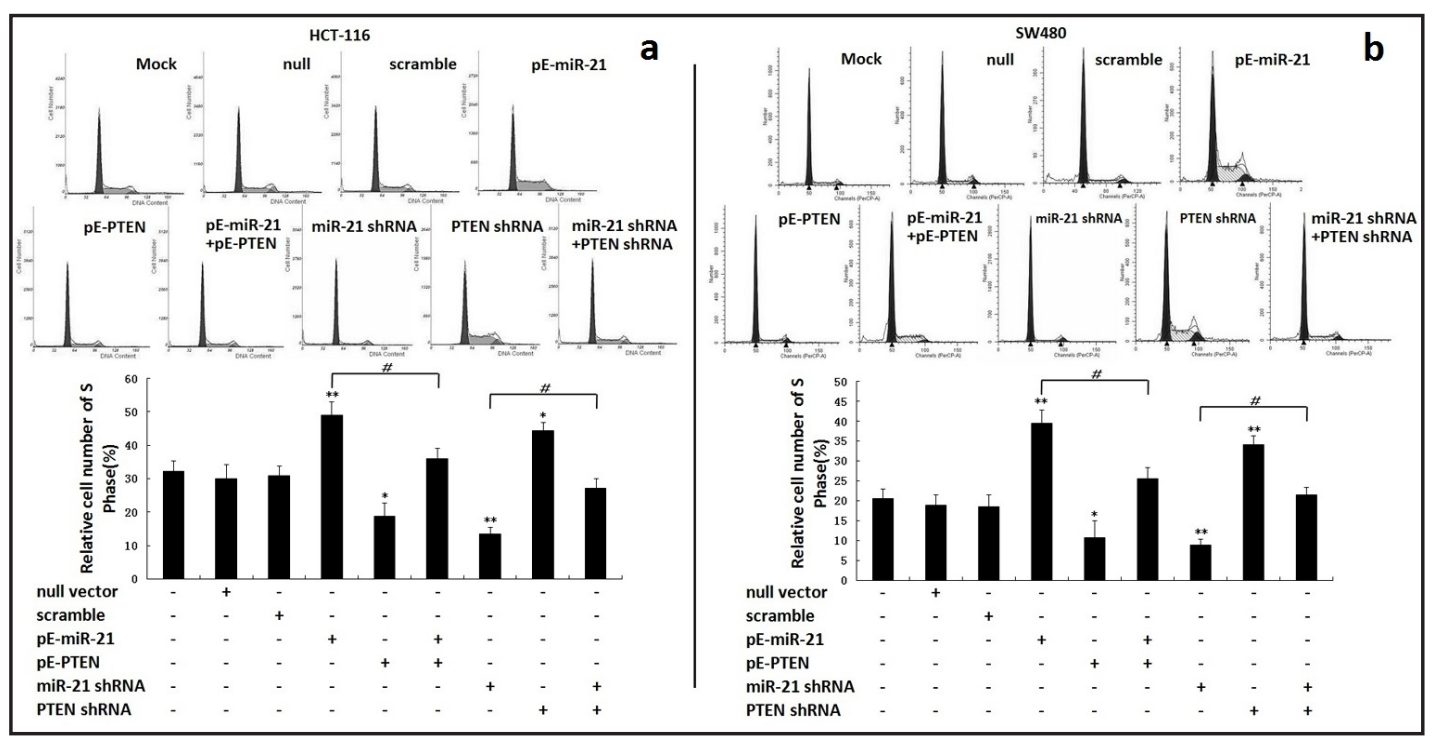

Fig. 6. miR-21 regulates cell cycle distribution of CRC cells through PTEN Recombinant plasmids were transfected alone or together into CRC cells. Flow cytometry of PI-stained cells was performed $48 \mathrm{~h}$ after transfection. Relative number of cells in S phase is shown in the histogram. (a) Cell cycle distribution of HCT-116 cells after transfection. (b) Cell cycle distribution of SW480 cells after transfection. All data are representative of three independent experiments. ${ }^{*} \mathrm{p}<0.05$, ${ }^{* *} \mathrm{p}<0.01$ vs blank control (Mock). ${ }^{*} \mathrm{p}<0.05$ vs cells transfected with pE-miR-21 or miR-21 shRNA.

miR-21 regulates cell cycle distribution and apoptosis in CRC cells through PTEN

To better understand the biological effects of miR-21 on CRC cells, we evaluated the cell cycle distribution and apoptosis using flow cytometry. As shown in Fig. 5, a decrease in apoptosis was observed in both HCT-116 and SW480 cells transfected with pE-miR-21, and an increase was observed in response to miR-21 shRNA. Meanwhile, an increase in the population of cells in S phase was observed for both cell lines transfected with pE-miR-21, and a decrease was observed in response to miR-21 shRNA (Fig. 6).

To further investigate whether miR-21 regulates cell cycle distribution and apoptosis in CRC cells by down-regulating PTEN, pE-miR-21- or miR-21 shRNA-transfected cells were co-transfected with pE-PTEN or PTEN shRNA. As shown in Fig. 5 and Fig. 6, the decrease in apoptosis and increase in the proportion of cells in S phase by pE-miR-21 were partially reversed by the over-expression of PTEN with pE-PTEN. In contrast, the increase in apoptosis and decrease in the proportion of cells in S phase by miR-21 shRNA were partially reversed by the knockdown of PTEN via its shRNA. These data indicated that miR-21 might accelerate cell cycle progression and inhibit apoptosis of CRC cells by down-regulating PTEN.

\section{miR-21 regulates invasion of CRC cells through PTEN}

The Chemicon cell invasion assay was employed to examine the effect of miR-21 on the invasiveness of CRC cells. The results clearly indicated that over-expression of miR-21 with pE-miR-21 in CRC cells could promote invasion. In contrast, inhibition of miR-21 activity with miR-21 shRNA led to a significant decrease in motility of CRC cells (Fig. 7). Furthermore, we conducted co-transfection experiments to examine whether miR-21 affects cellular invasion by regulating PTEN in CRC cells. We observed that silencing of PTEN with shRNA inhibited the effect of miR-21 shRNA and led to an increase in motility of CRC cells, and over-expression of PTEN with pE-PTEN inhibited the effect of pE-miR-21 treatment and led to a decrease in cell motility. These results suggested that miR-21 promoted the invasiveness of CRC cells by down-regulating PTEN. 


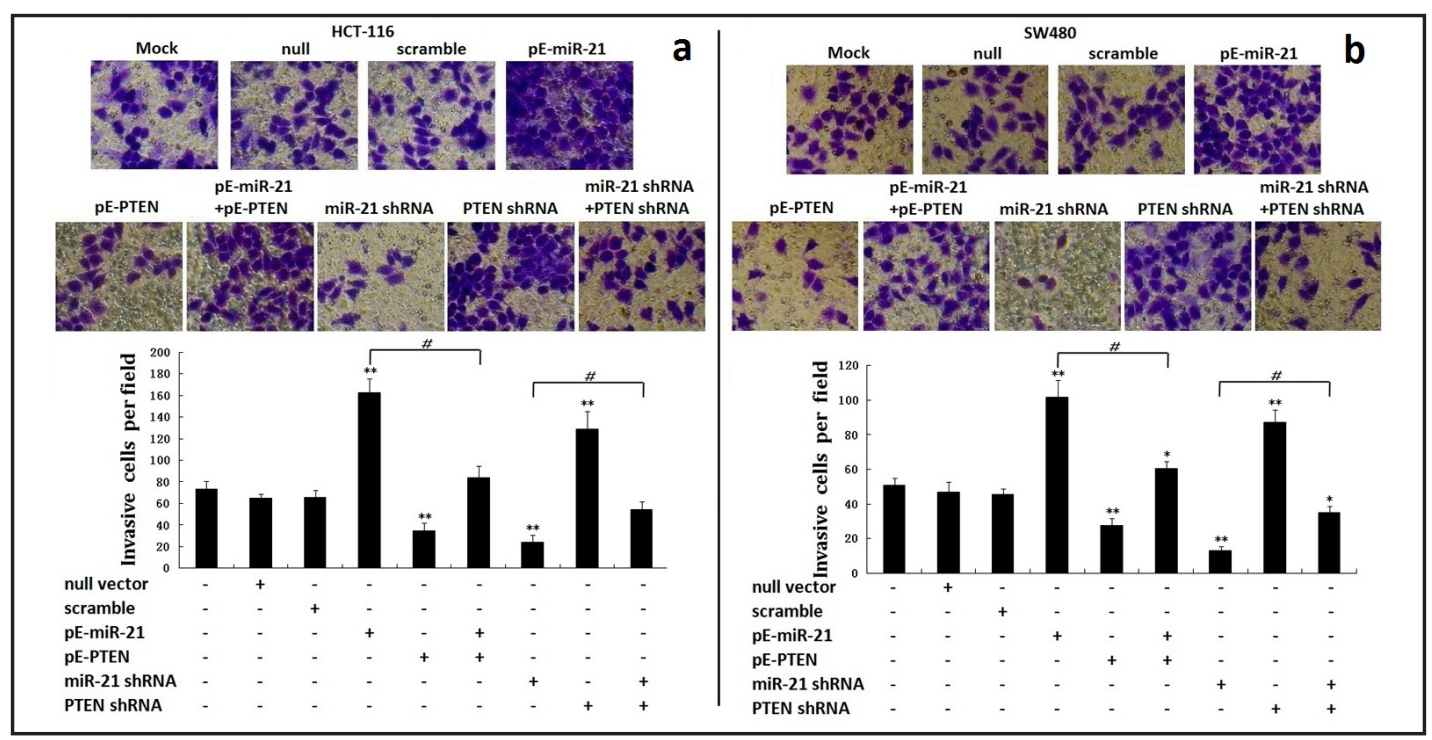

Fig. 7. miR-21 regulates invasiveness of CRC cells through PTEN Recombinant plasmids were transfected alone or together into CRC cells. Matrigel invasion assay was performed $48 \mathrm{~h}$ after transfection. Images of representative fields for each treatment were captured with a camera connected to a microscope (400x). Quantification data were obtained by counting the average numbers of cells from five different fields for each treatment. Average number of invasive cells per field for each treatment is shown in the histogram. (a) Change in the invasive ability of HCT-116 cells after transfection. (b) Change in the invasive ability of SW480 cells after transfection. All data are presented as the mean \pm SD of three independent experiments. ${ }^{*} \mathrm{p}<0.05$, ${ }^{* *} \mathrm{p}<0.01$ vs blank control (Mock). ${ }^{*} \mathrm{p}<0.05$ vs cells transfected with $\mathrm{pE}-\mathrm{miR}-21$ or miR-21 shRNA.

miR-21 modulates the growth of CRC xenografts

As miR-21 regulates the growth of CRC cells in vitro, we further assessed its effect on tumor formation in vivo. After selection in antibiotics for four months, stable clones were obtained. The expression of miR-21 was found to be increased or decreased in the stable clones transfected with pE-miR-21 or miR-21 shRNA as shown in Fig. 8c. The implanted tumors were noticeable 7 days after injection. Compared with the negative group, overexpression miR-21 significantly promoted tumor growth. Conversely, suppression of miR-21 markedly reduced tumor size (Fig. 8a) and tumor weight (Fig. 8b).

\section{Discussion}

miRNAs are small, noncoding RNAs that can negatively regulate gene expression at the translational level by base pairing with the 3' untranslated region (3'-UTR) of target mRNAs. With the increase in the number of studies on miRNAs, the aberrant expression of miRNAs has been identified in different types of cancer. miRNAs can function as tumor suppressors or oncogenes and regulate numerous important cancer-related genes. Over-expression of miRNAs can result in the down-regulation of tumor suppressor genes, whereas silencing of miRNAs can lead to the up-regulation of oncogenes [4,5]. Recently, a large number of miRNAs have been reported to be dysregulated in CRC and to affect tumor growth, migration and invasion by regulating certain genes [30-34].

miR-21 is one of the most prominent miRNAs implicated in the genesis and progression of human malignancy. Its expression is notably up-regulated in many cancers, including CRC, lung cancer, glioblastoma, hepatocellular carcinoma, and gastric cancer. In addition, its elevated expression level has been causally associated with tumor aggressiveness and poor prognosis [17-19, 24, 25, 35-39]. Numerous reports have demonstrated that miR-21 acts as a potential oncogene in CRC by promoting tumorigenesis, invasion and metastasis. For 


\section{Cellular Physiology

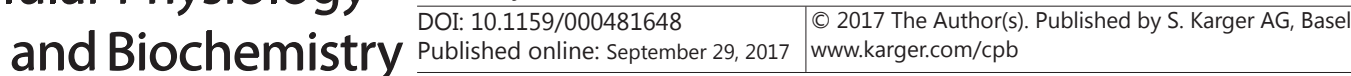 \\ Wu et al.: Mir-21 and Colorectal Cancer}

Fig. 8. miR-21 promotes tumor growth of HCT-116 cells subcutaneously implanted into nude mice (a) Growth curve of xenograft tumors. Tumor volume was measured every other day and used to construct the tumor growth curve. (b) Weight of xenograft tumors. Transplanted tumors were excised and weighed 28 days after inoculation. (c) Expression of miR-21 in stably transfected of HCT-116 cells. The expression of miR-21 in stable clones transfected with pE-miR-21, miR21 shRNA, Mock, null and scramble was examined using real-time PCR. Results were normalized to U6 expression and expressed as fold change relative to those in Mock. All data are presented as the mean \pm SD of three independent experiments. ${ }^{*} \mathrm{p}<0.05$, ${ }^{* *} \mathrm{p}<0.01$ vs negative control or Mock.

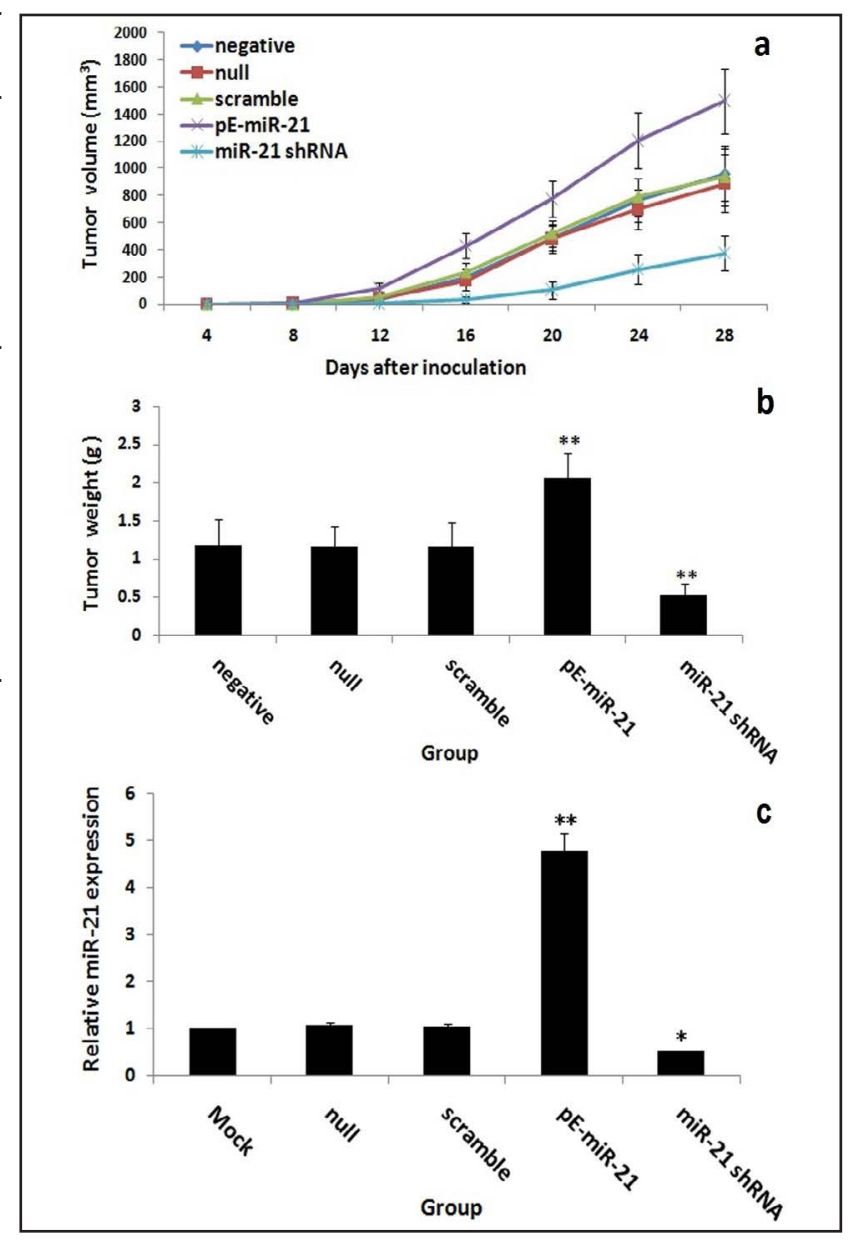

example, Yamamichi et al. have found that miR-21 expression was much higher in CRC tissue than that in normal mucosa. Meanwhile, significantly elevated expression of miR-21 has been observed in precancerous adenomas but not in nontumorigenic polyps. It has been indicated that miR-21 expression progressively increases during CRC progression from precancerous adenoma to advanced carcinoma [35]. In addition, the high expression of miR-21 and its association with advanced clinical stage, lymph node positivity and distal metastasis in CRC have been demonstrated in several studies [36-38]. Upon further study of the biological effects of miR-21, Liu et al. indicated that over-expression of miR-21 significantly promotes proliferation and invasion and inhibits apoptosis in human CRC cells, which may suggest its function as an oncogene in CRC [26]. In agreement with previous reports, our findings have shown that miR-21 is significantly up-regulated in CRC and is associated with advanced clinical TNM stage, poor differentiation, deeper invasion, positive lymph node metastasis and high level of serum CEA. Because our clinical data suggested that miR-21 was related to the progression of $\mathrm{CRC}$, we further investigated its biological function by transfecting CRC cells with ectopic-expression or shRNA-expression plasmids of miR-21. The results indicated that miR-21 could promote cell proliferation, accelerate cell cycle progression, inhibit apoptosis, and induce invasion of CRC cell lines. We further confirmed that miR-21 promotes the growth of CRC xenografts in nude mice in vivo. These findings suggested that miR-21 might promote malignant phenotypes in CRC, and hence it can be used as a potential biomarker for early diagnosis and treatment of CRC.

The tumor suppressor PTEN has been shown to be frequently mutated in a large number of human cancers, including CRC [28]. It has been reported that PTEN may function as a dual specificity phosphatase and regulate cell growth, apoptosis, invasion and differentiation by negatively regulating the PI3K/AKT signaling pathway. PTEN can 
down-regulate the level of lipid second messenger phosphoinositol 3, 4,5-triphosphate (PIP3) through dephosphorylation and subsequently suppress the activation of AKT/PKB, which mediates cell survival by regulating multiple target proteins involved in apoptosis. When PTEN is deleted, mutated, or inactivated, the activation of the PI3K/AKT signaling pathway can be triggered by numerous endogenous and exogenous stimuli, and this can promote the development of cancer. This pathway is known to play a key role in numerous cellular functions including proliferation, adhesion, angiogenesis, migration, invasion, and metabolism [40-42]. Further, loss of PTEN expression has been indicated to be significantly associated with advanced TNM stage, distant metastasis and poor prognosis in CRC [28, 29].

PTEN has also been reported as a direct target of miR-21, and it has been shown to be involved in miR-21-mediated cell proliferation, apoptosis inhibition, and invasion in human hepatocellular carcinoma [24], non-small cell lung cancer (NSCLC) [17], gastric cancer [18], endometrial cancer [19] and nasopharyngeal carcinoma [20]. Moreover, Xiong et al. observed a significant inverse correlation between miR-21 and PTEN protein in CRC tissues and cells and demonstrated that PTEN was a direct target of miR-21 in CRC through dual-luciferase reporter assays [16]. Our study showed that PTEN protein expression was significantly reduced in CRC tumor tissues and negatively correlated with the expression of miR-21, which was consistent with the results of previous studies. Moreover, we found that miR-21 could decrease PTEN protein expression and increase Akt phosphorylation in CRC cells. Furthermore, for the first time, we demonstrated the down-regulation of PTEN protein by miR-21 in CRC xenografts in nude mice.

Although the oncogenic function of miR-21 and the regulation of PTEN in CRC have been known, there was no direct evidence to affirm whether or not the miR-21-mediated malignant phenotypes of CRC cells were caused by PTEN. A previous study established a "rescue method" in which an miRNA inhibitor and specific siRNAs against its target gene were co-transfected into cells to modulate the protein level of the target and thus determine whether the miRNA affects cell phenotypes by the direct regulation of its target [24]. On the basis of this strategy, we developed a method that could bidirectionally change the protein level of PTEN by co-transfecting different combinations of the ectopic-expression or shRNAexpression plasmids of miR-21 and PTEN into CRC cells as follows: pE-miR-21 + pE-PTEN and miR-21-shRNA + PTEN-shRNA. Then, we detected the alterations in cell phenotypes in the different groups. Through a series of in vitro experiments, we found that silencing or elevating PTEN protein expression could partially reverse the effect of miR-21 shRNA or pE-miR-21 on apoptosis, cell cycle distribution, and invasion of CRC cells. Together with the previous findings, these results suggest that miR-21 might play an oncogenic role in the proliferation, apoptosis, cell cycle distribution and invasion of CRC cells by reducing PTEN protein levels and subsequently elevating Akt phosphorylation.

In conclusion, miR-21 plays an oncogenic role in the development and progression of CRC. It modulates malignant phenotypes such as proliferation, anti-apoptosis, cell cycle progression and invasion in CRC cells by down-regulating PTEN protein expression. Therefore, these data can help us understand the molecular mechanisms of miR-21 in CRC and provide valuable insights into the clinical use of miR-21 as a biomarker in this type of cancer.

\section{Acknowledgements}

This study was supported by the National Natural Science Foundation of China (NO. 81503090) and Natural Science Foundation of the education department of Sichuan Provinec (NO. 15ZA0260).

\section{Disclosure Statement}

The authors declare that they have no conflict of interest. 


\section{Cellular Physiology Cell Physiol Biochem 2017;43:945-958 and Biochemistry Published \begin{tabular}{l|l} 
DOI: 10.1159/000481648 & $\begin{array}{l}\text { C } 2017 \text { The Author(s). Published by S. Karger AG, Basel } \\
\text { www.karger.com/cpb }\end{array}$
\end{tabular}}

Wu et al.: Mir-21 and Colorectal Cancer

\section{References}

1 Siegel RL, Miller KD, Jemal A: Cancer statistics, 2015 CA Cancer J Clin 2015;65:5-29.

-2 Scheer A, Auer RA: Surveillance after curative resection of colorectal cancer. Clin Colon Rectal Surg 2009;22:242-250.

-3 Van Cutsem E, Nordlinger B, Cervantes A, Group EGW: Advanced colorectal cancer: ESMO Clinical Practice Guidelines for treatment. Ann Oncol 2010;21:v93-97.

4 Carthew RW, Sontheimer EJ: Origins and Mechanisms of miRNAs and siRNAs. Cell 2009;136:642-655.

5 Jansson MD, Lund AH: MicroRNA and cancer. Mol Oncol 2012;6:590-610.

6 Li HT, Zhang H, Chen Y, Liu XF, Qian J: MiR-423-3p enhances cell growth through inhibition of p21Cip1/ Waf1 in colorectal cancer. Cell Physiol Biochem 2015;37:1044-1054.

7 Xie T, Huang M, Wang Y, Wang L, Chen C, Chu X: MicroRNAs as Regulators, Biomarkers and Therapeutic Targets in the Drug Resistance of Colorectal Cancer. Cell Physiol Biochem 2016;40:62-76.

-8 Han R, Sun Q Wu J, Zheng P, Zhao G: Sodium Butyrate Upregulates miR-203 Expression to Exert AntiProliferation Effect on Colorectal Cancer Cells. Cell Physiol Biochem 2016;39:1919-1929.

-9 Xuan Y, Yang H, Zhao L, Lau WB, Lau B, Ren N, Hu Y, Yi T, Zhao X, Zhou S, Wei Y: MicroRNAs in colorectal cancer: small molecules with big functions. Cancer Lett 2015;360:89-105.

10 Liu X, Xie T, Mao X, Xue L, Chu X, Chen L: MicroRNA-149 Increases the Sensitivity of Colorectal Cancer Cells to 5-Fluorouracil by Targeting Forkhead Box Transcription Factor FOXM1 Cell Physiol Biochem 2016;39(2):617-29

11 Stintzing S, Lenz HJ: MicroRNA-21 in colorectal cancer: “Just another brick in the wall”? J Natl Cancer Inst 2013;105:840-841.

12 Xia X, Yang B, Zhai X, Liu X, Shen K, Wu Z, Cai J: Prognostic role of microRNA-21 in colorectal cancer: a meta-analysis. PLoS One 2013;8:e80426.

13 Li T, Leong MH, Harms B, Kennedy G, Chen L: MicroRNA-21 as a potential colon and rectal cancer biomarker. World J Gastroenterol 2013;19:5615-5621.

$14 \mathrm{Xu} \mathrm{J}$, Zhang W, Lv Q, Zhu D: Overexpression of miR-21 promotes the proliferation and migration of cervical cancer cells via the inhibition of PTEN. Oncol Rep 2015;33:3108-3116.

15 Qin X, Yan L, Zhao X, Li C, Fu Y: microRNA-21 overexpression contributes to cell proliferation by targeting PTEN in endometrioid endometrial cancer. Oncol Lett 2012;4:1290-1296.

16 Xiong B, Cheng Y, Ma L, Zhang C: MiR-21 regulates biological behavior through the PTEN/PI-3 K/Akt signaling pathway in human colorectal cancer cells. Int J Oncol 2013;42:219-228.

17 Liu ZL, Wang H, Liu J, Wang ZX: MicroRNA-21 (miR-21) expression promotes growth, metastasis, and chemo- or radioresistance in non-small cell lung cancer cells by targeting PTEN. Mol Cell Biochem 2013;372: 35-45.

18 Zhang BG, Li JF, Yu BQ Zhu ZG, Liu BY, Yan M: microRNA-21 promotes tumor proliferation and invasion in gastric cancer by targeting PTEN. Oncol Rep 2012;27:1019-1026.

-19 Qin X, Yan L, Zhao X, Li C, Fu Y: microRNA-21 overexpression contributes to cell proliferation by targeting PTEN in endometrioid endometrial cancer. Oncol Lett 2012;4:1290-1296.

-20 Ou H, Li Y, Kang M: Activation of miR-21 by STAT3 induces proliferation and suppresses apoptosis in nasopharyngeal carcinoma by targeting PTEN gene. PLoS One 2014;9:e109929.

-21 Li X, Xin S, He Z, Che X, Wang J, Xiao X, Chen J, Song X: MicroRNA-21 (miR-21) post-transcriptionally downregulates tumor suppressor PDCD4 and promotes cell transformation, proliferation, and metastasis in renal cell carcinoma. Cell Physiol Biochem 2014;33:1631-1642.

22 Li X, Xin S, He Z, Che X, Wang J, Xiao X, Chen J, Song X: MicroRNA-21 (miR-21) post-transcriptionally downregulates tumor suppressor PDCD4 and promotes cell transformation, proliferation, and metastasis in renal cell carcinoma. Cell Physiol Biochem 2014;33:1631-1642.

23 Peacock O, Lee AC, Cameron F, Tarbox R, Vafadar-Isfahani N, Tufarelli C, Lund JN: Inflammation and MiR-21 pathways functionally interact to downregulate PDCD4 in colorectal cancer. PLoS One 2014;9:e110267.

24 Liu C, Yu J, Yu S, Lavker RM, Cai L, Liu W, Yang K, He X, Chen S: MicroRNA-21 acts as an oncomir through multiple targets in human hepatocellular carcinoma. J Hepatol 2010;53:98-107.

25 Lei BX, Liu ZH, Li ZJ, Li C, Deng YF: miR-21 induces cell proliferation and suppresses the chemosensitivity in glioblastoma cells via downregulation of FOXO1 Int J Clin Exp Med 2014;7:2060-2066. 


\section{Cellular Physiology Cell Physiol Biochem 2017;43:945-958 \begin{tabular}{l|l|l} 
DOI: 10.1159/000481648 & $\begin{array}{l}\text { O 2017 The Author(s). Published by S. Karger AG, Basel } \\
\text { www.karger.com/cpb }\end{array}$ \\
\hline
\end{tabular} \\ Wu et al.: Mir-21 and Colorectal Cancer}

26 Liu M, Tang Q, Qiu M, Lang N, Li M, Zheng Y, Bi F: miR-21 targets the tumor suppressor RhoB and regulates proliferation, invasion and apoptosis in colorectal cancer cells. FEBS Lett 2011;585:2998-3005.

-27 Fu YR, Liu XJ, Li XJ, Shen ZZ, Yang B, Wu CC, Li JF, Miao LF, Ye HQ, Qiao GH, Rayner S, Chavanas S, Davrinche C, Britt WJ, Tang Q, Mcvoy M, Mocarski E, Luo MH: MicroRNA miR-21 attenuates human cytomegalovirus replication in neural cells by targeting Cdc25a. J Virol 2015;89:1070-1082.

28 Sun Y, Tian H, Wang L: Effects of PTEN on the proliferation and apoptosis of colorectal cancer cells via the phosphoinositol-3-kinase/Akt pathway. Oncol Rep 2015;33:1828-1836.

-29 Papadatos-Pastos D, Rabbie R, Ross P, Sarker D: The role of the PI3K pathway in colorectal cancer. Crit Rev Oncol Hematol 2015;94:18-30.

30 Wei W, Yang Y, Cai J, Cui K, Li RX, Wang H, Shang X, Wei D: MiR-30a-5p Suppresses Tumor Metastasis of Human Colorectal Cancer by Targeting ITGB3 Cell Physiol Biochem 2016;39:1165-1176.

-31 Zhou L, Xu Z, Ren X, Chen K, Xin S: MicroRNA-124 (MiR-124) Inhibits Cell Proliferation, Metastasis and Invasion in Colorectal Cancer by Downregulating Rho-Associated Protein Kinase 1(ROCK1). Cell Physiol Biochem 2016;38:1785-1795.

-32 Zhang W, Zou C, Pan L, Xu Y, Qi W, Ma G, Hou Y, Jiang P: MicroRNA-140-5p inhibits the progression of colorectal cancer by targeting VEGFA. Cell Physiol Biochem 2015;37:1123-1133.

33 Xu K, Liu X, Mao X, Xue L, Wang R, Chen L, Chu X: MicroRNA-149 suppresses colorectal cancer cell migration and invasion by directly targeting forkhead box transcription factor FOXM1 Cell Physiol Biochem 2015;35:499-515.

34 Fang Y, Sun B, Xiang J, Chen Z: MiR-301a promotes colorectal cancer cell growth and invasion by directly targeting SOCS6 Cell Physiol Biochem 2015;35:227-236

-35 Yamamichi N, Shimomura R, Inada K, Sakurai K, Haraguchi T, Ozaki Y, Fujita S, Mizutani T, Furukawa C, Fujishiro M, Ichinose M, Shiogama K, Tsutsumi Y, Omata M, Iba H: Locked nucleic acid in situ hybridization analysis of miR-21 expression during colorectal cancer development. Clin Cancer Res 2009;15:4009-4016.

-36 Nielsen BS, Jorgensen S, Fog JU, Sokilde R, Christensen IJ, Hansen U, Brunner N, Baker A, Moller S, Nielsen HJ: High levels of microRNA-21 in the stroma of colorectal cancers predict short disease-free survival in stage II colon cancer patients. Clin Exp Metastasis 2011;28:27-38.

-37 Lee KS, Nam SK, Koh J, Kim DW, Kang SB, Choe G, Kim WH, Lee HS: Stromal Expression of MicroRNA-21 in Advanced Colorectal Cancer Patients with Distant Metastases. J Pathol Transl Med 2016;50:270-277

38 Xiong Y, Zhang YY, Wu YY, Wang XD, Wan LH, Li L, Zhou LM: Correlation of over-expressions of miR-21 and Notch-1 in human colorectal cancer with clinical stages. Life Sci 2014;106:19-24.

39 Zhou X, Wang X, Huang Z, Wang J, Zhu W, Shu Y, Liu P: Prognostic value of miR-21 in various cancers: an updating meta-analysis. PLoS One 2014;9:e102413.

40 Bunney TD, Katan M: Phosphoinositide signalling in cancer: beyond PI3K and PTEN. Nat Rev Cancer 2010;10:342-352.

41 Chen J, Lin C, Yong W, Ye Y, Huang Z: Calycosin and genistein induce apoptosis by inactivation of HOTAIR/pAkt signaling pathway in human breast cancer MCF-7 cells. Cell Physiol Biochem 2015;35:722-728.

42 Chen J, Duan Y, Zhang X, Ye Y, Ge B, Chen J: Genistein induces apoptosis by the inactivation of the IGF-1R/pAkt signaling pathway in MCF-7 human breast cancer cells. Food Funct 2015;6:995-1000. 